\section{JTI}

JOURNAL OF

TRAUMA AND INJURY

Received: May 8, 2020

Revised: June 1, 2020

Accepted: June 26, 2020

\section{Correspondence to}

\section{Jae Hun Kim, M.D.}

Department of Trauma Surgery and Surgical Critical Care, Pusan National University Hospital, 179 Gudeok-ro, Seo-gu, Busan 49241, Korea

Tel: $+82-51-240-7369$

Fax: $+82-51-240-7719$

E-mail:drtrauma73@gmail.com

\title{
Blunt Transection of the Entire Anterolateral Abdominal Wall Musculature Following Seatbelt- Related Injury
}

\author{
Hohyun Kim, M.D. ${ }^{1,2,3}$, Jae Hun Kim, M.D. ${ }^{1,2,4}$, Gil Hwan Kim, M.D. ${ }^{1,2}$, \\ Hyun-Woo Sun, M.D. ${ }^{1,2}$, Chan Ik Park, M.D. ${ }^{1,2}$, Sung Jin Park, M.D. ${ }^{1,2}$, \\ Chan Kyu Lee, M.D. ${ }^{1,2}$, Suk Kim, M.D. ${ }^{2,5,6}$ \\ ${ }^{1}$ Department of Trauma Surgery and Surgical Critical Care, Pusan National University \\ Hospital, Busan, Korea \\ ${ }^{2}$ Biomedical Research Institute, Pusan National University Hospital, Busan, Korea \\ ${ }^{3}$ Department of Emergency Medicine, Pusan National University School of Medicine, \\ Yangsan, Korea \\ ${ }^{4}$ Department of General Surgery, Pusan National University School of Medicine, Yangsan, \\ Korea \\ ${ }^{5}$ Department of Diagnostic Radiology, Pusan National University Hospital, Busan, Korea \\ ${ }^{6}$ Department of Diagnostic Radiology, Pusan National University School of Medicine, \\ Yangsan, Korea
}

Traumatic abdominal wall hernias (TAWHs) are uncommon and the incidence of this, which is rarely encountered in clinical practice, has been estimated at $1 \%$. Furthermore, blunt transection of the entire abdominal wall musculature caused by seatbelt is a very rare complication. We report a case of adult with a complete disruption of the entire anterolateral abdominal wall muscle following the seatbelt injury. A 32-year-old male was wearing a seat belt in a high speed motor vehicle collision. Abdominal computed tomography (CT) scan revealed the complete disruption of bilateral abdominal wall musculatures including TAWH without visceral injury. However, injuries of small bowel and sigmoid colon were observed in the intra-operative field. The patient underwent the repair by primary closure of the defect with absorbable monofilament sutures. This case suggests that especially in TAWH patients, even if a CT scan is normal, clinicians should keep the possibility of bowel injury in mind, and choose a treatment based on the clinical findings.

Keywords: Hernia, Abdominal; Seat belts; Abdominal muscles; Herniorrhaphy; Multiple trauma 


\section{INTRODUCTION}

A traumatic abdominal wall hernia (TAWH) is defined as a bowel or abdominal organ herniation through a disruption of the musculature and fascia after trauma [1]. TAWH is rare, occurring after a low- or high-velocity impact of a blunt object on the abdominal wall, constituting approximately $1 \%$ of all blunt injuries [1,2]. In motor vehicle collisions, the wearing of a seatbelt is associated with certain abdominal injuries. A seatbelt seldom causes injury. However, in those with high-energy seatbelt injuries, lumbar hernias are common. A pediatric case exhibiting complete transection of all of the anterolateral abdominal wall muscles (the rectus abdominis, external oblique, internal oblique, and transversus abdominis muscles) by the seatbelt has been reported [3]. However, TAWHs featuring complete abdominal wall transection caused by a seatbelt are rare in adults [4].

Here, we report the case of an adult with a TAWH caused by a seatbelt accompanied by complete transection of all anterolateral abdominal wall muscles; we briefly review the literature.

\section{CASE REPORT}

A 32-year-old male with no significant past medical history was involved in a high-speed road traffic accident while wearing a seatbelt. He arrived at our emergency room in stable condition. He was assessed using the Advanced Trauma Life Support protocol. He complained of pain in the lower abdomen and left leg. His Glasgow Coma Scale score was 15 at presentation and his vital signs were within normal limits. He was unable to fully move his left leg (his femur was fractured). He was slightly obese (body mass index [BMI], $31.2 \mathrm{~kg} / \mathrm{m}^{2}$ ). The abdomen exhibited a pannus with seatbelt marks, bruising, and skin maceration over the lower abdomen and pelvis. Otherwise, the abdomen was soft and the lower abdomen exhibited moderate tenderness and swelling.

A contrast-enhanced computed tomography (CECT) scan of the abdomen and pelvis revealed a large muscular defect involving the entire lower abdominal wall, and subcutaneous visceral herniation of the small intestine and the colon (Fig. 1). The seatbelt had caused full-thickness disruption of the entire anterolateral abdominal wall
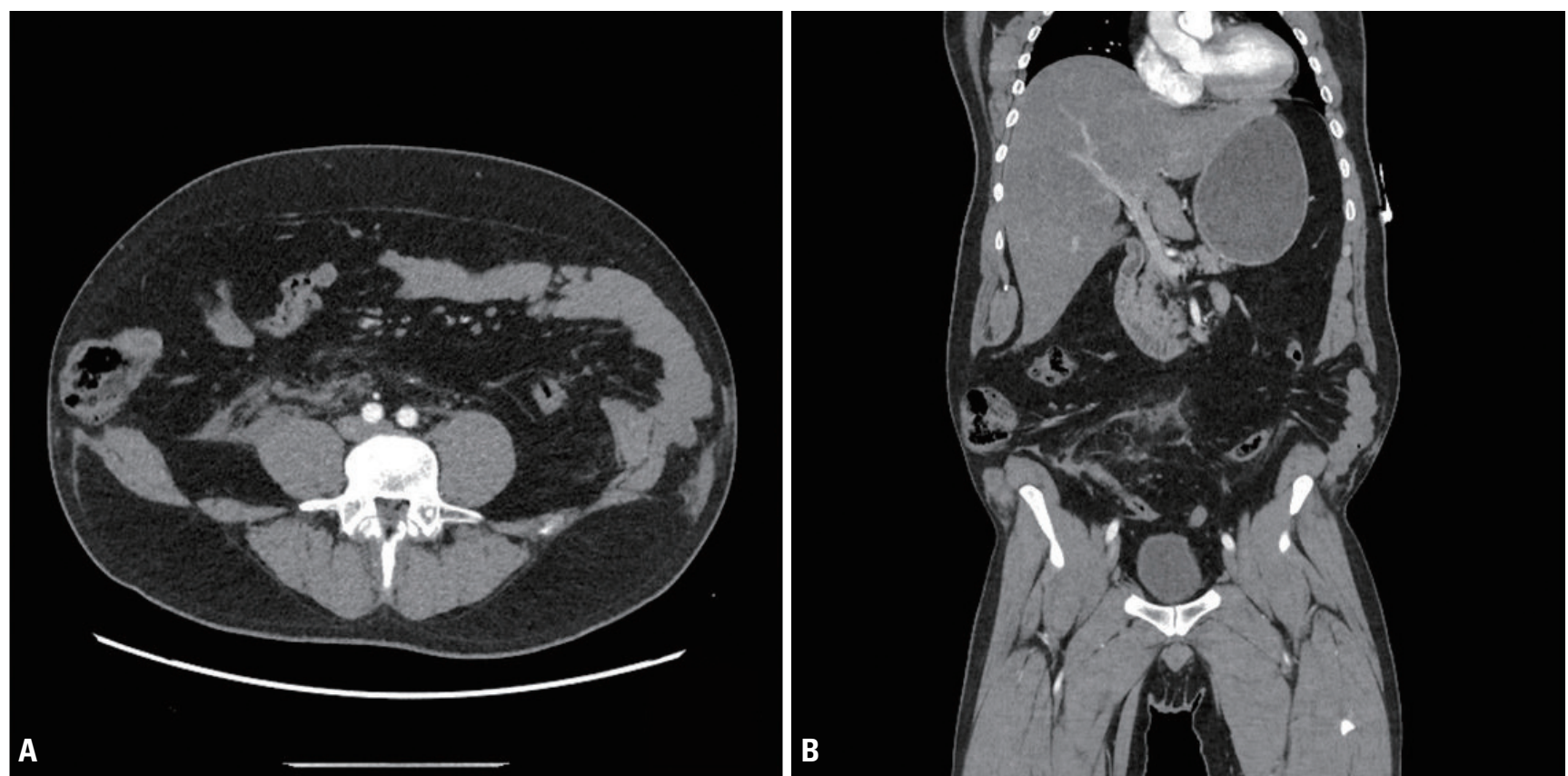

Fig. 1. Initial, abdominal computed tomography (CT) findings. Axial (A) and coronal (B) abdominal CT scans reveal injury to the bilateral external oblique and internal oblique muscles, and a traumatic abdominal wall hernia. No active bleeding was noted. 
including the rectus abdominis, external oblique, internal oblique, and transversus abdominis muscles, extending bilaterally to the posterior elements. Subcutaneous fat stranding was evident over the lower abdomen but no active bleeding was noted. The liver and spleen were unremarkable. Pelvic imaging revealed a small amount of blood and fat stranding. No definitive evidence of hollow viscus injury as free peritoneal air was apparent in CT.

We performed exploratory laparotomy through a midline incision because patient had high- energy injuries and high probability of having associated intra-abdominal injuries. In exploratory laparotomy, approximately $500 \mathrm{~mL}$
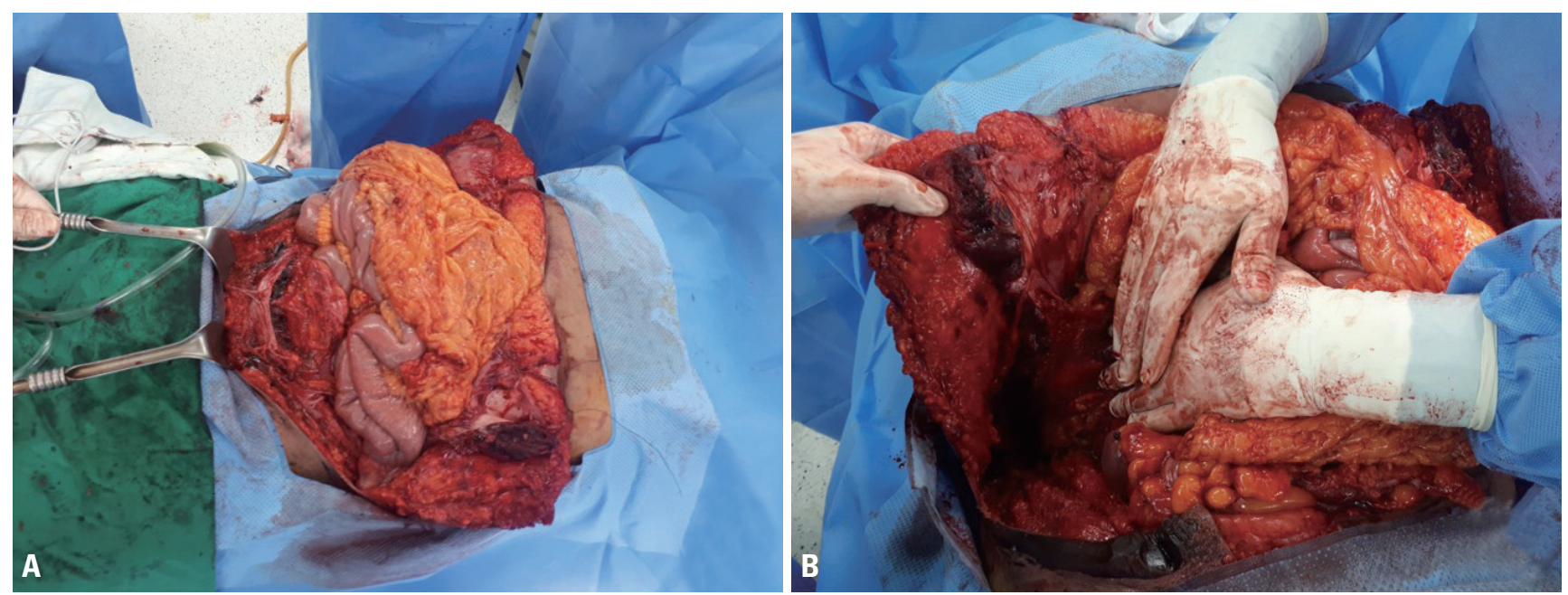

Fig. 2. The operative field. Intra-operative photographs reveal (A) complete disruption of the entire anterior abdominal wall musculature, (B) including the rectus, external and internal oblique, and transversus abdominis muscles, extending bilaterally to the posterior elements.
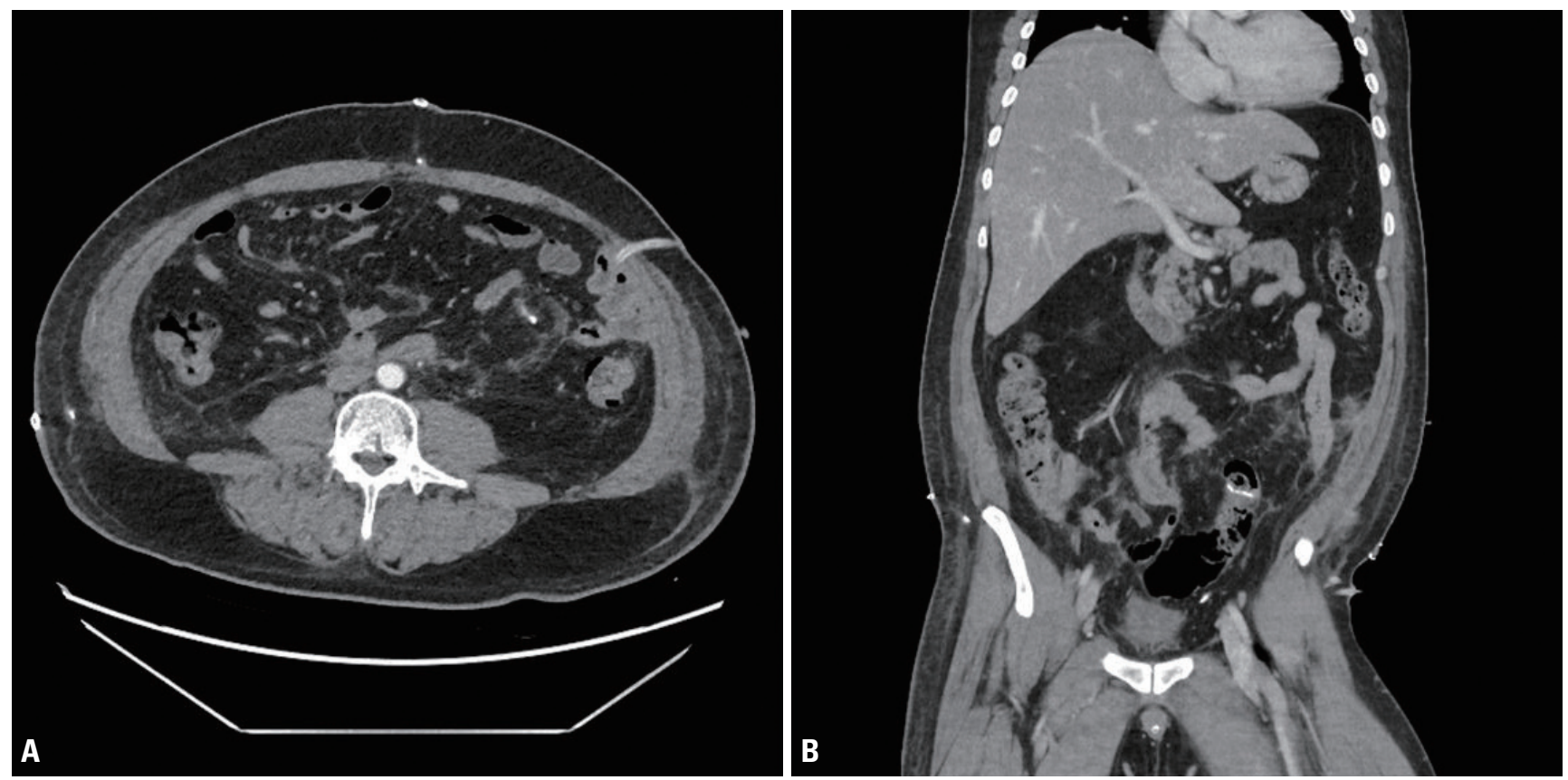

Fig. 3. Abdominal computed tomography (CT) findings on postoperative day 7. Neither the axial (A) nor coronal (B) abdominal CT scan reveals any evidence of an abdominal wall hernia or any other complication. 
intraperitoneal blood was found. Mesenteric avulsions of the small bowel and sigmoid colon, with wide serosal disruption, were also apparent, requiring resection and anastomosis. In addition, the bilateral rectus abdominis, external oblique, internal oblique, and transverse abdominal muscles were completely disrupted, with herniation of the small bowel and the colon (Fig. 2).

We performed abdominal wall exploration via a separate transverse incision. The superior and inferior epigastric vessels, the deep circumflex iliac vessels, and branches of the mesenteric vessels, were transected. However, no active bleeding was evident; thrombi blocked the blood vessels. The abdominal wall muscles were repaired in a layer-by-layer manner using interrupted monofilament absorbable sutures. On postoperative day 7, follow-up CECT revealed no evidence of herniation or any other complication (Fig. 3). Photographic finding on postoperative day 21 showed an inverted T-shape incision wound (Fig. 4). The patient recovered after prolonged hospitalisation caused by a need for rehabilitation and a pressure sore on his left heel. He was discharged on postoperative day 51 without any clinical evidence of a hernia.

\section{DISCUSSION}

The abdominal wall is divided into anterior, lateral, and posterior compartments [5]. The rectus abdominis

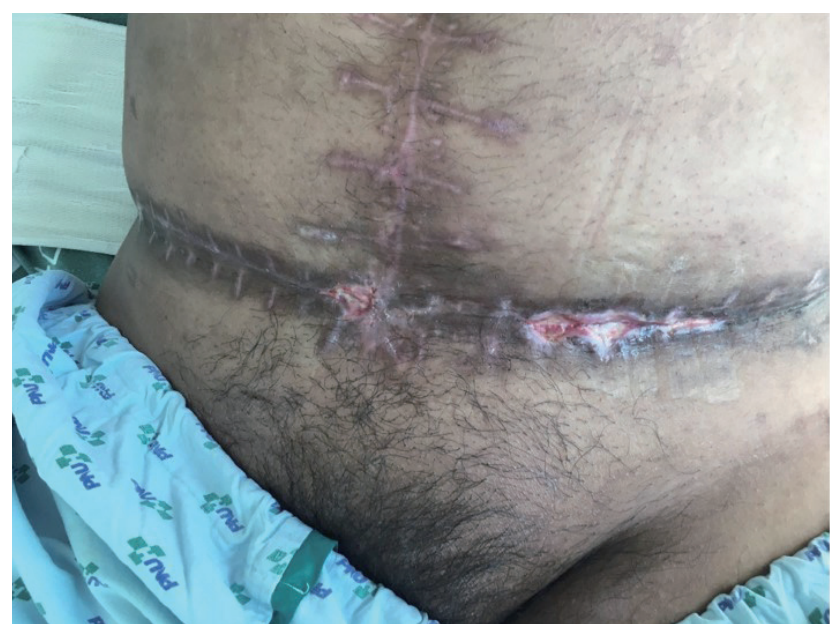

Fig. 4. Postoperative wound on postoperative day 21. Photograph shows an inverted T-shape incision wound. muscles constitute the anterior compartment. The lateral compartment contains the external oblique, internal oblique, and transversus abdominis muscles (both superficial and deep). The posterior compartment contains the latissimus dorsi, quadratus lumborum, and erector spinae (paraspinal) muscles. Fig. 5 demonstrate the normal cross-sectional imaging of the abdominal wall.

TAWH is defined as disruption of muscles and the fascia without skin penetration after blunt abdominal trauma. TAWH is rare ( $1 \%$ of all blunt abdominal traumas, which are common $[1,2])$. Motor vehicle collisions cause most (60-70\%) TAWHs [6-8]; 46-48\% of patients with TAWHs had been wearing seatbelts $[6,8]$. Injury is caused by a sudden increase in intra-abdominal pressure and application of extensive shear forces to the abdominal wall. TAWH is typically located at anatomically weak areas of the lower abdomen; lumbar hernias are the most common type of TAWH [5]. Complete transection of the abdominal wall musculature is rare. Our case, however, exhibited TAWH featuring complete transection of the anterolateral abdominal wall musculature by the seatbelt.

Seatbelts probably increase the risk of TAWH, because they exert a direct compressive force on the abdominal cavity. Patients of high BMI may be at particular risk for

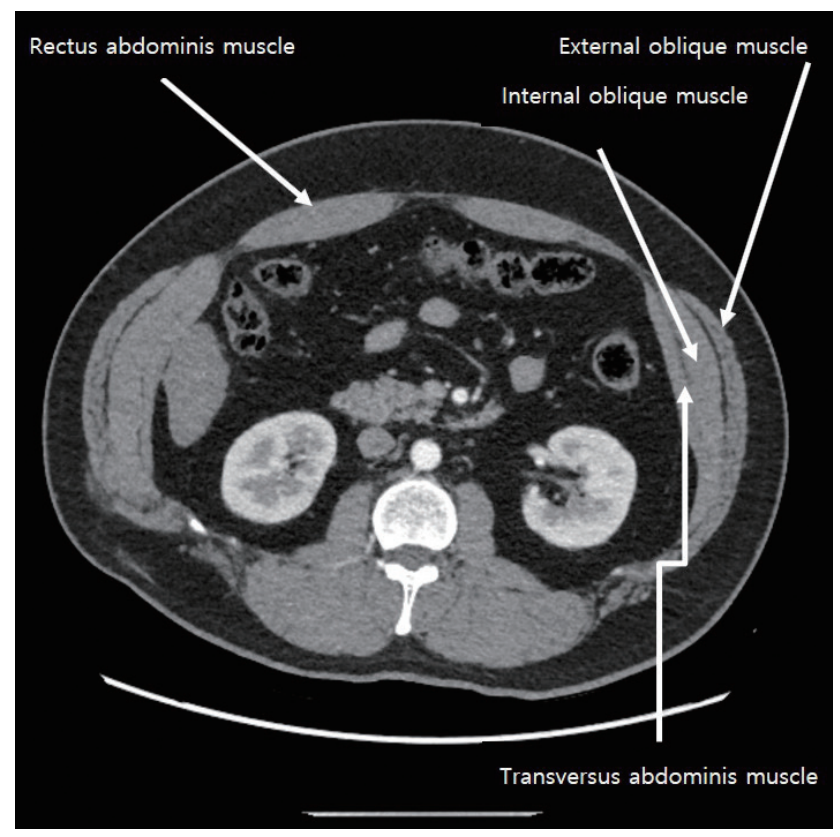

Fig. 5. Normal cross-sectional imaging of the abdominal wall. 
TAWH [9]; such patients often wear seatbelts incorrectly [9]. Incorrect placement of a seatbelt across the abdomen rather than the bony pelvis may cause shearing of abdominal muscles from their attachments along the pelvic brim. Alternatively, the seatbelt may cause a sudden increase in intra-abdominal pressure triggering rupture of the abdominal wall [9]. A seatbelt is often worn incorrectly by the obese [10]. Thus, a high index of suspicion for TAWH is required for overweight and obese patients, particularly those involved in motor vehicle crashes while wearing seatbelts.

The "seatbelt lethal triad," a term coined by Kulvatunyou et al. [11], is a triad of injuries associated with the use of three-point seatbelts, and includes TAWH, hollow viscus injury, and abdominal vascular injury. Our present case had TAWH and a hollow viscus injury. The superior and inferior epigastric vessels, deep circumflex iliac vessels, and branches of the mesenteric vessels were transected, but bleeding was not observed because the vessels were occluded by thrombi.

TAWHs can be difficult to diagnose; the clinical manifestations are equivocal. In physical examinations, focal tenderness and ecchymosis of the abdominal wall may be apparent, sometimes accompanied by a visible bulge. Contusions of the anterior abdominal wall under the seatbelt (the "seatbelt sign") may also be evident, and should raise the index of concern in the context of TAWH and intra-abdominal visceral and vascular injuries. In some studies, concurrent intra-abdominal injuries were found in approximately $60-90 \%$ of patients with TAWHs $[7,8,12,13]$. More than $50 \%$ of TAWHs cannot be detect-

Table 1. Abdominal wall disruption grades $[2,13]$

\begin{tabular}{|ll|}
\hline $\begin{array}{c}\text { Abdominal wall } \\
\text { injury grade }\end{array}$ & \multicolumn{1}{c|}{ Definition } \\
\hline I & Subcutaneous tissue contusion \\
\hline III & Abdominal wall hematoma \\
IV & Single abdominal wall muscle disruption \\
VI & Complete abdominal wall muscle disruption \\
& $\begin{array}{c}\text { Complete abdominal wall muscle disruption } \\
\text { with herniation of abdominal contents }\end{array}$ \\
\hline
\end{tabular}

ed by physical examination, being identified only via CT $[7,8]$. The utilities of conventional radiology and barium contrast films are debatable; these modalities are rarely used. CT is the most accurate TAWH diagnostic tool and should also be used to detect associated intra-abdominal injuries $[1,12,14]$. A simple CT-based grading system for abdominal wall disruption has been developed (Table 1) $[2,13]$. According to this system, the injury of our patient was of grade $\mathrm{V}$.

However, it is not easy to distinguish a hernia that is not bleeding from a previous non-traumatic hernia. Therefore, a traumatic hernia should be carefully sought, based on both the characteristics of the injury and the clinical findings, even if a CT scan is normal, particularly in patients with high-energy injuries.

The therapeutic approach varies greatly; patient-tailored (case-by-case) management is required $[12,15]$. Although no clear consensus on the timing or nature of surgical repair has emerged, several factors must be considered, including defect size, the contents of the hernia, and associated injuries, with special attention being paid to the bowel [5]. If a trauma patient is stable and the hernia is small, with visceral organs protruding through the defect, exploratory laparotomy and/or laparoscopy should be urgently performed to prevent possible visceral incarceration. If the abdominal wall defect is large and urgent surgical exploration of the abdomen is not indicated, repair can be delayed, allowing the skin to heal and recovery from any associated injuries [15]. In our case, as the abdominal wall defect was large and the TAWH was not associated with active bleeding or other intra-abdominal injury (as revealed by initial abdominal CT), the choice of treatment was difficult. However, bowel injury was possible; the patient had suffered a high-energy injury that completely ruptured the abdominal wall muscles. We chose early repair, and found other abdominal injuries (to the small intestine and colon) intra-operatively. Thus, immediate surgical repair with primary tissue closure was in fact appropriate.

In conclusion, complete transection of the entire anterolateral abdominal wall musculature (the rectus abdominis, external oblique, internal oblique, and transversus abdominis muscles) is rare. However, seatbelt injuries may include complete rupture of this musculature result- 
ing in subcutaneous bowel herniation. The case presented here emphasises the importance of a high index of clinical suspicion in the context of TAWH and early radiological evaluation, such as CT, to minimise complications. Especially in TAWH patients, even if a CT scan is normal, clinicians should keep the possibility of bowel injury in mind, and choose a treatment based on the clinical findings. Also, seatbelts and/or seatbelt should be worn in the correct manner to prevent seatbelt-related injuries.

\section{ACKNOWLEDGEMENTS}

This work was supported by clinical research grant from Pusan National University Hospital in 2020.

\section{REFERENCES}

1. Damschen DD, Landercasper J, Cogbill TH, Stolee RT. Acute traumatic abdominal hernia: Case reports. J Trauma 1994;36:273-6.

2. Dennis RW, Marshall A, Deshmukh H, Bender JS, Kulvatunyou $\mathrm{N}$, Lees JS, et al. Abdominal wall injuries occurring after blunt trauma: Incidence and grading system. Am J Surg 2009;197:4137.

3. Moremen JR, Nakayama DK, Ashley DW, Astin M, Nolan TL. Traumatic disruption of the abdominal wall: lap-belt injuries in children. J Pediatr Surg 2013;4:e21-4.

4. Patel K, Doolin R, Suggett N. Blunt transection of rectus abdominis following seatbelt related trauma with associated small and large bowel injury. Int J Surg Case Rep 2013;4:958-60.

5. Matalon SA, Askari R, Gates JD, Patel K, Sodickson AD, Khu- rana B. Don't forget the abdominal wall: Imaging spectrum of abdominal wall injuries after nonpenetrating trauma. Radiographics 2017;37:1218-35.

6. Coleman JJ, Fitz EK, Zarzaur BL, Steenburg SD, Brewer BL, Reed RL, et al. Traumatic abdominal wall hernias: location matters. J Trauma Acute Care Surg 2016;80:390-6; discussion 396-7.

7. Netto FA, Hamilton P, Rizoli SB, Nascimento B Jr, Brenneman FD, Tien H, et al. Traumatic abdominal wall hernia: epidemiology and clinical implications. J Trauma 2006;61:1058-61.

8. Honaker D, Green J. Blunt traumatic abdominal wall hernias: associated injuries and optimal timing and method of repair. J Trauma Acute Care Surg 2014;77:701-4.

9. Gutteridge I, Towsey K, Pollard C. Traumatic abdominal wall herniation: case series review and discussion. ANZ J Surg 2014;84:160-5.

10. Pardhan A, Mazahir S, Rao S, Weber D. Blunt traumatic abdominal wall hernias: a surgeon's dilemma. World J Surg 2016;40:231-5.

11. Kulvatunyou N, Bender JS, Albrecht RM. Traumatic abdominal wall hernia classification. J Trauma Acute Care Surg 2013;75:536.

12. Liasis L, Tierris I, Lazarioti F, Clark CC, Papaconstantinou HT. Traumatic abdominal wall hernia: is the treatment strategy a real problem? J Trauma Acute Care Surg 2013;74:1156-62.

13. Suhardja TS, Atalla MA, Rozen WM. Complete abdominal wall disruption with herniation following blunt injury: case report and review of the literature. Int Surg 2015;100:531-9.

14. Pickett D, Geiger J, Leskovan JJ, Moore J, Stausmire JM. Lapbelt-induced pediatric blunt traumatic abdominal wall hernia. Am Surg 2017;83:e189-91.

15. Al Beteddini OS, Abdulla S, Omari O. Traumatic abdominal wall hernia: a case report and literature review. Int J Surg Case Rep 2016;24:57-9. 